\title{
Tratamento da síndrome respiratória aguda severa causada pelo vírus Sars-Cov-2 com bloqueadores dos receptores de angiotensina II
}

\author{
Treatment of severe acute respiratory syndrome caused by Sars-Cov-2 virus with \\ Angiotensin II receptor blockers
}
Tratamiento del síndrome respiratorio agudo severo causado por el virus Sars-Cov-2 con bloqueadores de los receptores de angiotensina II

Ana Flávia Oliveira Pampolha ${ }^{1 *}$, Maria Helena Rodrigues de Mendonça ${ }^{1,2}$, Elem Cristina Rodrigues Chaves², Bruna Verônica Azevedo Gois'

\section{RESUMO}

Objetivo: Realizar uma revisão sobre o COVID-19 e um possível tratamento com Bloqueadores dos Receptores de Angiotensina II (BRA's) em pacientes que desenvolveram a síndrome respiratória aguda severa. Métodos: Estudo de revisão da literatura nas bases PubMed, Medline, Scielo, Elsevier e Google acadêmico, de 2000 a 2020 a partir dos descritores: "coronavírus", "tratamento", "anti-hipertensivo" e "síndrome respiratória aguda severa". Resultados: A partir da busca literária da patologia, epidemiologia e biologia molecular da COVID-19 e farmacologia dos BRA's, 49 trabalhos foram lidos completamente e, ao final, um total de 31 artigos foi selecionado para contemplar o estudo, sendo estes classificados de acordo com o tema, autor/ano e tipo de estudo, sendo 71\% (22) publicado em 2020 e 3,2\% (1) aos demais anos. Dentre os temas abordados, $25,8 \%$ (8) abordam a "COVID-19/Sars-CoV-2 e os aspectos clínicos e moleculares", seguido de "Cytokine Storm e a resposta imune" (16,1\%). Considerações finais: Essa revisão bibliográfica agrega informações que indicam os benefícios do uso de BRAs no tratamento da Síndrome Respiratória Aguda Severa causada pelo COVID-19, portanto, vão a consenso as diretrizes no sentido a não suspensão dos BRAs no curso de infecção pela COVID-19. Contudo, comprovação da eficácia do fármaco requer experimentos laboratoriais mais específicos.

Palavras-chave: COVID-19, Síndrome respiratória aguda severa, Bloqueadores do receptor tipo 1 de angiotensina II.

\section{ABSTRACT}

Objective: To review COVID-19 and possible treatment with Angiotensin II receptor blockers (ARB's) in patients who developed severe acute respiratory syndrome. Methods: Review study of the literature in the databases PubMed, Medline, Scielo, Elsevier and Google academic, from 2000 to 2020 based on the descriptors: "coronavirus", "treatment", "antihypertensive" and "severe acute respiratory syndrome". Results: From the literary search of the pathology, epidemiology and molecular biology of COVID-19 and pharmacology of the ARBs, 49 works were read completely and, at the end, a total of 31 articles were selected to contemplate the study, being these classified according to the theme, author/year and type of study, being $71 \%$ (22) published in 2020 and $3.2 \%$ (1) to other years. Among the topics covered, $25.8 \%$ (8) approach "COVID 19/Sars-Cov-2 and the clinical and molecular aspects", followed by "Cytokine Storm and the immune response" (16.1\%). Final considerations: This bibliographic review aggregates information that indicates the benefits of using Bras in the treatment of severe acute respiratory syndrome caused by COVID-19, therefore, there is consensus on the guidelines for not suspending Bras in the course of COVID-19 infection. However, proving the efficacy of the drug requires more specific laboratory experiments.

Keywords: COVID-19, Severe acute respiratory syndrome, Angiotensin II type 1 receptor blockers.

\footnotetext{
1 Universidade do Estado do Pará (UEPA), Belém - PA. *E-mail: ana.flaviapampolha@gmail.com

2 Centro Universitário Fibra, Belém - PA.
} 


\section{RESUMÉN}

Objetivo: Realizar una revisión sobre el COVID-19 y un posible tratamiento com bloqueadores de los receptores de angiotensina II (BRA's) en pacientes que desarrollaron el síndrome respiratorio agudo severo. Métodos: Estudio de revisión de la literatura en las bases PubMed, Medline, Scielo, Elsevier y Google académico, de 2000 a 2020 a partir de los descritores: "coronavirus", "tratamiento", "antihipertensivo" y "síndrome respiratorio agudo severo". Resultados: A partir de la búsqueda literária de la patología, epidemiología y biología molecular de COVID-19 y la farmacología de los BRAs, 49 trabajos fueron leídos completamente y, al final, un total de 31 artículos fue seleccionado para contemplar el estudio, siendo éstos clasificados de acuerdo con el tema, autor/año y tipo de estudio, siendo $71 \%$ (22) publicado en 2020 y 3,2\% (1) a los demás años. Entre los temas abordados, 25,8\% (8) abordan la "COVID-19/Sars-CoV-2 y los aspectos clínicos y moleculares", seguido de "Cytokine Storm y la respuesta inmune" (16,1\%). Consideraciones finales: Esta revisión bibliográfica agrega información que indica los beneficios del uso de bras en el tratamiento del Síndrome Respiratorio Agudo Severo causado por el COVID19, por lo tanto, van a consenso las directrices en el sentido de no suspensión de los bras en el curso de infección por la COVID-19. Sin embargo, la comprobación de la eficacia del fármaco requiere experimentos de laboratorio más específicos.

Palabras clave: COVID-19, Síndrome respiratorio agudo severo, Bloqueadores del receptor tipo 1 de angiotensina II.

\section{INTRODUÇÃO}

Os coronavírus (CoV) pertencem à família Coronaviridae, vírus envelopados de RNA fita simples, não segmentado e sentido positivo, foi estruturalmente reconhecido por microscopia eletrônica por suas glicoproteínas transmembranas distintas denominadas spike (S) que se projetam em formato de coroa (WALLS AC, et al., 2020). Além disso, trata-se de uma doença zoonótica que pode ser transmitida entre humanos pelo ar e infecta as vias aéreas superiores e inf eriores causando sintomas semelhantes aos da gripe comum, com probabilidade de evoluir para sintomas neurológicos, entéricos e, principalmente, a Síndrome Respiratória Aguda Severa, conhecida pela sigla em inglês SARS (WALLS AC, et al., 2020; ADHIKARI SP, et al., 2020).

Desde dezembro de 2019, foi detectada uma serie de casos de pneumonia emergentes na província de Wuhan, na China, que foram posteriormente associados ao novo coronavírus, denominado Sars-CoV-2, causador da doença pelo coronavírus 2019 (COVID-19) (LANA RM, et al., 2020). Apesar de haver uma baixa taxa de mortalidade e a maioria dos pacientes desenvolverem sintomas brandos, ainda há uma quantidade preocupante de indivíduos em situação crítica e de óbitos (YE Q, et al., 2020).

Nesse cenário, a pandemia ultrapassa os valores combinados de mortalidade causados pela primeira epidemia de SARS entre 2002 e 2003 e pela Síndrome Respiratória do Oriente Médio (MERS), do ano de 2013 (HUANG C, et al., 2020; BAVICHI C, et al., 2020). De acordo com os dados divulgados pelo Ministério da Saúde, até julho de 2021, houve desde o início da pandemia 19.797.086 casos de Síndrome Respiratória Aguda Severa e 553.179 mortes causadas pelo vírus Sars-CoV-2 no Brasil (MINISTÉRIO DA SAÚDE, 2021).

Destarte, ao adentrar as vias aéreas superiores, o novo coronavírus interage com o receptor da enzima conversora de angiotensina 2 (rECA2) por meio de suas proteínas de superfície "spike" onde, segundo a literatura, o SARS-CoV-2 tem maior afinidade com o receptor do ECA2 em comparação ao SARS-CoV-1, responsável pela primeira epidemia de SARS. A partir disso, a interação vírus-receptor permite a disseminação do vírus por todo o sistema respiratório (RIVELLESE F e PREDILETTO E, 2020).

Contextualizando, a enzima conversora de angiotensina 2 (ECA2) pertence ao sistema reninaangiotensina (SRA) e é responsável por balanceá-lo ao degradar a angiotensina II (Ang II) - cuja função no SRA é aumentar a pressão arterial, a permeabilidade celular e promover o processo de inflamação e proliferação celular - em angiotensina (1-7) e angiotensina I - precursora da Ang II - em angiotensina (1-9). (MARIA MLD, 2015; UZUNIAN A, 2020).

Após a infecção viral, as proteínas ECA2 sofrem downregulation, ou seja, o decréscimo da proteína na membrana celular - diretamente por conta da própria ligação com o vírus e indiretamente por conta da lise 
celular. Com isso, ocorre um desequilíbrio na homeostase no sistema cardiovascular uma vez que a angiotensina II perde seu "freio" e permanece ativa, e em excesso, interage com o seu respectivo receptor do tipo I de angiotensina II (rAT1) (UZUNIAN A, 2020). Em sequência, a proteína Angiotensina II induz a ativação local de células do sistema imune, e por conta da ativação exacerbada do SRA, ocorre o consequente evento denominado cytokine storm (RIVELLESE F e PREDILETTO E, 2020; ZHANG P, et al., 2020).

O termo "cytokine storm" se refere a uma severa reação imune ocasionada pela liberação descontrolada de citocinas, proteínas sinalizadoras, na corrente sanguínea. Nesse aspecto, dentre as muitas funções das citocinas, estão o controle da proliferação e diferenciação celular e a regulação da angiogênese e das respostas imunológicas e inflamatórias (TISONCIK JR, et al. 2012; UZUNIAN A, 2020).

Nesse contexto, estudos recentes têm mostrado que o aumento de citocinas pro-inflamatórias no soro sanguíneo está associado com a inflamação pulmonar e com o extenso dano tecidual no pulmão causado em pacientes infectados com COVID-19 com Síndrome Respiratória Aguda Severa (BRANDÃO SCS, et al., 2020; HUANG C, et al., 2020).

Outrossim, as citocinas interleucinas (IL) IL-1b, IL-2, IL-7, IL-8, IL-9, IL-10, IL-17, o Fator Estimulador de Colônia de Granulócitos (em inglês, "Granulocyte colony-stimulating factor" ou G-CSF), Fator Estimulante de Colônia de Macrófagos de Granulócitos (em inglês, "Granulocyte-macrophage colony-stimulating factor" ou GM-CSF), Interferon gamma (IFNg), Fator de Necrose Tumoral Alfa (TNFa), citocina quimiotática chamada Monócitos Quimiotáticos Proteína-1 (MCP1), quimiocinas Proteína 10 Indutível (IP10) e as Proteínas Inflamatórias do Macrófago 1A e 1B (em inglês, "Macrophage Inflammatory Proteins" ou MIP1A e MIP1B) foram descritas como as principais agravantes nesse processo e podem levar o paciente a óbito (BRANDÃO SCS, et al., 2020; WU D e YANG XO, 2020).

A angiotensina II também induz a proliferação de linfócitos T e células "Natural Killer" (NK) e a liberação de fatores de transcrição, tais como o Fator Nuclear-kappa B (NF-kB), Proteína Ativadora 1 (AP-1) e o Fator Induzido pela Hipóxia do tipo 1(HIF-1) (KASAL D e NEVES M, 2011). Além disso, há um "cross-talk" entre os fatores NF-kB - principal família pró-inflamatória - e a família HIF - que possui importante papel regulador durante os casos de hipóxia enquanto o fator AP-1 - composto porproteínas ativadoras - tem seu mecanismo dependente de NF-kB (FUJIOKA S, et al., 2004; D'IGNAZIO L, et al., 2016).

Ademais, o sistema renina-angiotensina também está diretamente conectado ao sistema imune, haja vista que os próprios linfócitos e macrófagos expressam receptores rAT1 e os linfócitos Te as células NK possuem seu próprio sistema funcional de renina-angiotensina, o que indica que os sistemas locais existem e podem servir para aumentar os ef eitos da Angll circulante e seu papel no processo de inflamação (JUREWICZ M, et al., 2007; ZHANG J e CROWLEY SD, 2013).

Apesar de ser o menos letal que os coronavírus associados à SARS e MERS, o SARS-CoV-2, é o mais contagioso e a elevada taxa de hospitalização resultante do COVID-19 tem causado o colapso do sistema de saúde em muitos países aumentando, portanto, a necessidade de um medicamento eficaz com baixa toxicidade para tratar as infecções (CARVALHO CA, et al., 2020; MEO SA, et al., 2020).

Nesse sentido, o inibidor da enzima de conversão da angiotensina (IECA) - que inibe a formação da angiotensina II -, os bloqueadores dos receptores de angiotensina II (BRA's) - que antagoniza seletivamente os receptores de angiotensina II - e os betabloqueadores adrenérgicos, todos esses fármacos antihipertensivos, representam uma possível alternativa no tratamento da doença ou na prevenção do desenvolvimento de casos severos (MANCIA, et al., 2020).

Um estudo observacional analítico concluiu que dentre os pacientes com hipertensão que foram internados com COVID-19, o tratamento hospitalar com IECA/BRA's esteve associado com o menor risco de mortalidade por todas as causas em comparação com os não usuários desses anti-hipertensivos (ZHANG J, et al., 2020). Outro estudo semelhante avaliou que, de acordo com os dados obtidos, houve uma modesta redução na porcentagem de casos positivos para COVID-19 entre pacientes que fazem tratamento com betabloqueadores, o que sugere que esse achado pode estar associado aos ef eitos dos betabloqueadores na expressão ou apresentação dos receptores de ECA2 na superfície celular (REYNOLDS HR, et al., 2020). 
Diante disso, este trabalho teve como objetivo realizar uma revisão sobre o COVID-19 e um possível tratamento com os Receptores de Angiotensina em pacientes que desenvolveram a Síndrome Respiratória Aguda Severa.

\section{MÉTODOS}

Trata-se de em estudo de revisão integrativa da literatura nas bases de dados PubMed, Medical Literature Analysis and Retrieval System Online (MEDLINE), Scientific Eletronic Library Online (SCIELO), Elsevier e Google acadêmico, no período entre 2000 e 2020. Os principais descritores em saúde utilizados foram "coronavírus", "tratamento", "anti-hipertensivo" e "síndrome respiratória aguda severa" e suas correspondentes em inglês "coronavirus", "treatment", "antihypertensive" e "SARS".

Foram estabelecidos como critérios de exclusão: artigos publicados antes de 2000 ou que se referiam somente a outros tipos de síndromes respiratórias não causadas pela infecção pelos vírus da família Coronaviridae; como critérios de inclusão: trabalhos nas modalidades de artigo científico, teses e relatórios técnicos de pesquisa disponíveis em sua totalidade em concordância com a temática do estudo, indexados nos idiomas português e inglês no período de 2000 até 2020 .

Após a leitura de título, foram excluídos os que não diziam respeito ao objetivo do estudo e, seguindo a leitura dos estudos, foram selecionados 31 artigos que preenchiam os critérios de inclusão e, portanto, condizentes com a proposta da pesquisa (Figura 1).

Figura 1 - Fluxograma metodológico referente as etapas de revisão da literatura.

\begin{tabular}{|c|c|c|c|}
\hline $\begin{array}{c}\text { Busca } \\
\text { bibliográfica em } \\
\text { plataformas de } \\
\text { bases de dados }\end{array}$ & $\begin{array}{l}\text { Uso de } \\
\text { descritores em } \\
\text { português e } \\
\text { inglês }\end{array}$ & $\begin{array}{l}\text { Determinação } \\
\text { dos critérios de } \\
\text { inclusão }\end{array}$ & $\begin{array}{l}\text { Determinação } \\
\text { dos critérios de } \\
\text { exclusão }\end{array}$ \\
\hline $\begin{array}{c}\text {-PubMed, Medical } \\
\text { Literature Analysis } \\
\text { and Retrieval System } \\
\text { Online (MEDLINE), } \\
\text { Scientific Eletronic } \\
\text { Library Online } \\
\text { (SCIELO), Elsevier e } \\
\text { Google acadêmico }\end{array}$ & $\begin{array}{c}\text { •"coronavírus"; } \\
\text { "tratamento"; "anti- } \\
\text { hipertensivo"; } \\
\text { "síndrome respiratória } \\
\text { aguda severa" e suas } \\
\text { correspondentes em } \\
\text { inglês "coronavirus"; } \\
\text { "treatment"; } \\
\text { "antihypertensive"; } \\
\text { "SARS". }\end{array}$ & $\begin{array}{l}\text { •Artigos nas } \\
\text { modalidades tese de } \\
\text { doutorado, artigo } \\
\text { científico, relatório } \\
\text { técnico de pesquisa } \\
\text { publicados no período } \\
\text { de } 2000 \text { à } 2020 \text {. }\end{array}$ & $\begin{array}{l}\text { •Artigos publicados } \\
\text { antes de } 2000 \text {, artigos } \\
\text { referentes a outras } \\
\text { síndromes } \\
\text { respiratórias não } \\
\text { relacionadas ao vírus } \\
\text { Sars-CoV }\end{array}$ \\
\hline
\end{tabular}

Fonte: Pampolha AFO, et al., 2021.

\section{RESULTADOS}

A partir da busca literária, 49 trabalhos foram lidos completamente e, ao final, um total de 31 artigos foram selecionados para contemplar o estudo, sendo estes classificados de acordo com o tema, autor/ano e tipo de estudo, sendo 22 dos trabalhos publicados em 2020 e 9 nos demais anos. Dentre os temas abordados, 8 trabalhos se enquadram no tema "COVID-19/Sars-CoV-2 e os aspectos clínicos e moleculares", 2 em "Comparação entre COVID-19, SARS e MERS", 4 no tema "Relação entre a angiotensina II(ECA2) e COVID 19", 5 no tema "Cytokine Storm e a resposta imune", 3 estão inclusos em "Anti-hipertensivos /Bloqueadores de receptor da ANG II", 4 em "Hipertensão/inflamação e Angiotensina II" e 2 no tema "Relação entre o sistema renina-angiotensina (SRA) e a idade" (Quadro 1). 
Quadro 1 - Caracterização do acervo de revisão, segundo autor, ano (2000-2020) e a respectiva metodologia adotada.

\begin{tabular}{|c|c|c|c|c|}
\hline Tema & Autor/Ano & Título & Tipo de Estudo & Principais Resultados \\
\hline \multirow{8}{*}{$\begin{array}{l}\text { COVID-19/Sars-CoV-2 e os } \\
\text { aspectos clínicos e moleculares }\end{array}$} & Wang L, et al., 2019 & $\begin{array}{l}\text { Review of the } 2019 \text { novel coronavins } \\
\text { (SARS-CoV-2) based on current } \\
\end{array}$ & Revisão & $\begin{array}{l}\text { Resumo sistemático da epidemiologia, características clínicas, } \\
\text { diagnóstico, tratamento e a prevenção da COVID-19. } \\
\end{array}$ \\
\hline & $\begin{array}{l}\text { Carvalho CA, et al., } \\
\qquad 2020\end{array}$ & $\begin{array}{l}\text { In Vitro Inhibition of SARS-CoV-2 Infection } \\
\text { by Bovine Lactoferrin }\end{array}$ & Experimental & $\begin{array}{l}\text { Sugere que a inibição do Sars-CoV-2 pela lactoferrina bovina tem } \\
\text { o potencial de constituir uma abordagem bioquímica para } \\
\text { combater a nova pandemia de coronavírus. }\end{array}$ \\
\hline & Huang C, et al., 2020 & $\begin{array}{l}\text { Clinical features of patients infected with } \\
2019 \text { novel coronavirus in Wuhan, China }\end{array}$ & $\begin{array}{l}\text { Observacional } \\
\quad \text { analítico }\end{array}$ & $\begin{array}{l}\text { A COVID-19 causou grupos de doenças respiratórias graves } \\
\text { semelhantes a doenças respiratórias agudas graves e foi } \\
\text { associada à admissão na UTI e alta mortalidade. }\end{array}$ \\
\hline & Shang J, et al., 2020 & $\begin{array}{l}\text { Structure of mouse coronavirus spike } \\
\text { protein complexed with receptor reveals } \\
\text { mechanism for viral entry }\end{array}$ & Experimental & $\begin{array}{l}\text { Além de seu papel bem caracterizado na ligação viral às células } \\
\text { hospedeiras, a ligação ao receptor também induz a mudança } \\
\text { conformacional da proteína spike resultando na fusão do vírus } \\
\text { com a célula hospedeira. }\end{array}$ \\
\hline & $\begin{array}{l}\text { Adhikari SP, et al., } \\
\qquad 2020\end{array}$ & $\begin{array}{l}\text { Epidemiology, causes, clinical } \\
\text { manifestation and diagnosis, prevention } \\
\text { and control of coronavirus disease } \\
\text { (COVID-19) during the early outbreak } \\
\text { period:a scoping review }\end{array}$ & Revisão & $\begin{array}{l}\text { Resumo sistemático da epidemiologia, características clínicas, } \\
\text { diagnóstico, tratamento e a prevenção da COVID-19. }\end{array}$ \\
\hline & Lana RM, et al., 2020 & $\begin{array}{l}\text { Emergência do novo coronavírus (SARS- } \\
\text { CoV-2) e o papel de uma vigilância } \\
\text { nacional em saúde oportuna e efetiva }\end{array}$ & Revisão & $\begin{array}{l}\text { Resumo sistemático da epidemiologia, características clínicas, } \\
\text { diagnóstico, tratamento e a prevenção da COVID-19. }\end{array}$ \\
\hline & Walls AC, et al., 2020 & $\begin{array}{l}\text { Structure, function, and antigenicity of the } \\
\text { SARS-CoV-2 spike glycoprotein }\end{array}$ & Experimental & $\begin{array}{l}\text { A microscopia crioeletrônica da glicoproteína spike do SARS- } \\
\text { CoV-2 fornecem um projeto para o design de vacinas e } \\
\text { terapêutica. }\end{array}$ \\
\hline & Yan H, et al., 2020 & $\begin{array}{l}\text { Role of Drugs used for chronic disease } \\
\text { management on Susceptibility and } \\
\text { Severity of COVID-19: A Large Case- } \\
\text { Control Study }\end{array}$ & Caso-Controle. & $\begin{array}{l}\text { Sem evidência que justifique alterar a terapia com BRA's ou IECA } \\
\text { no contexto da pandemia. Foi encontrado um risco aumentado de } \\
\text { COVID-19 em associação com inibidores da DPP4 e insulina e } \\
\text { risco reduzido com inibidores da glucosidase. }\end{array}$ \\
\hline \multirow[t]{2}{*}{$\begin{array}{l}\text { Comparação entre COVID-19, } \\
\text { SARS e MERS }\end{array}$} & Meo SA, et al., 2020 & $\begin{array}{l}\text { Novel coronavirus 2019-nCoV: } \\
\text { prevalence, biological and clinical } \\
\text { characteristics comparison with SARS- } \\
\text { CoV and MERS-CoV }\end{array}$ & $\begin{array}{l}\text { Pesquisa de } \\
\text { levantamento }\end{array}$ & $\begin{array}{l}\text { SARS-CoV é mais contagioso que MERS-CoV, porém com menor } \\
\text { taxa de mortalidade. }\end{array}$ \\
\hline & Petrosillo N, et al., 2020 & $\begin{array}{l}\text { COVID-19, SARS and MERS: are they } \\
\text { closely related? }\end{array}$ & $\begin{array}{l}\text { Pesquisa de } \\
\text { levantamento }\end{array}$ & $\begin{array}{l}\text { SARS-CoV } 2 \text { é mais contagioso que a MERS-CoV e o SARS- } \\
\text { CoV. }\end{array}$ \\
\hline $\begin{array}{l}\text { Relação entre a angiotensina } \\
\text { II(ECA2) e COVID-19 }\end{array}$ & $\begin{array}{l}\text { Reynolds HR, et al., } \\
\qquad 2020\end{array}$ & $\begin{array}{l}\text { Renin-angiotensin-aldosterone system } \\
\text { inhibitors and risk of Covid-19 }\end{array}$ & $\begin{array}{l}\text { Observacional } \\
\text { analítico }\end{array}$ & $\begin{array}{l}\text { Não encontramos nenhum aumento substancial na positividade } \\
\text { para Covid- } 19 \text { ou aumento de risco de Covid- } 19 \text { grave entre os } \\
\text { pacientes em associação com as cinco classes comuns de } \\
\text { medicamentos anti-hipertensivos analisados. }\end{array}$ \\
\hline
\end{tabular}




\begin{tabular}{|c|c|c|c|c|}
\hline Tema & Autor/Ano & Título & Tipo de Estudo & Principais Resultados \\
\hline & $\begin{array}{l}\text { Vaduganathan M, et al., } \\
2020\end{array}$ & $\begin{array}{l}\text { Renin-angiotensin-aldosterone system } \\
\text { inhibitors in patients with Covid-19 }\end{array}$ & $\begin{array}{l}\text { Observacional } \\
\text { descritivo }\end{array}$ & $\begin{array}{l}\text { Proteínas denominadas "spike" presentes nos coronavírus } \\
\text { possuem interação com os receptores ECA2, facilitando a entrada } \\
\text { do vírus no sistema respiratório. }\end{array}$ \\
\hline & Baral R, et al., 2020 & $\begin{array}{l}\text { Effect of renin-angiotensin-aldosterone } \\
\text { system inhibitors in patients with COVID- } \\
19: \text { a systematic review and meta-analysis } \\
\text { of } 28,872 \text { patients }\end{array}$ & Artigo de opinião & $\begin{array}{l}\text { Encontradas evidências dos efeitos benéficos do uso crônico de } \\
\text { IECA/BRA's, especialmente em pacientes em coorte hipertensiva } \\
\text { com COVID-19. }\end{array}$ \\
\hline & $\begin{array}{l}\text { Rivellese \& Prediletto, } \\
2020\end{array}$ & $\begin{array}{l}\text { ACE2 at the centre of COVID-19 from } \\
\text { paucisymptomatic infections to severe } \\
\text { pneumonia }\end{array}$ & Artigo de opinião & $\begin{array}{l}\text { Indica que a proteína ECA2 poderia ser a ligação direta entre a } \\
\text { infecção por SARS-CoV-2 e o desenvolvimento de lesão } \\
\text { pulmonar e hiperinflamação. }\end{array}$ \\
\hline \multirow{5}{*}{$\begin{array}{l}\text { Cytokine Storm e a resposta } \\
\text { imune }\end{array}$} & Fujioka S, et al., 2004 & $\begin{array}{l}\text { NF-kB and AP-1 connection: mechanism } \\
\text { of NF-kB-dependent regulation of AP-1 } \\
\text { activity }\end{array}$ & Experimental & $\begin{array}{l}\text { Ocorrência de cytokine storm em pacientes com COVID-19 em } \\
\text { casos de infecção grave. }\end{array}$ \\
\hline & $\begin{array}{l}\text { Tisoncik JR, et al., } \\
2012\end{array}$ & Into the eye of the cytokine storm & Revisão & $\begin{array}{l}\text { Aborda sobre os efeitos da citokine storm na patologia de } \\
\text { doenças clínicas e infecciosas e discute a dificuldade de usar o } \\
\text { conhecimento da citokine storme das terapias imunomoduladoras } \\
\text { para melhorar os resultados clínicos para pacientes com } \\
\text { infecções agudas graves. }\end{array}$ \\
\hline & D'Ignazio L, et al., 2016 & $\begin{array}{l}\text { NF-kB and HIF crosstalk in immune } \\
\text { responses }\end{array}$ & Revisão & $\begin{array}{l}\text { Discute o crosstalk entre HIF e NF-KB no controle do sistema } \\
\text { imune em diferentes células imunes como macrófagos, neutrófilos } \\
\text { e células B e T. }\end{array}$ \\
\hline & Ye Q, et al., 2020 & $\begin{array}{l}\text { The pathogenesis and treatment of the } \\
\text { "Cytokine Storm" in COVID-19 }\end{array}$ & Revisão & $\begin{array}{l}\text { Discute sobre os efeitos da citokine storm nos casos graves de } \\
\text { COVID-19. }\end{array}$ \\
\hline & Wu D \& Yang XO, 2020 & $\begin{array}{l}\text { TH17 responses in cytokine storm of } \\
\text { COVID-19: An emerging target of JAK2 } \\
\text { inhibitor Fedratinib }\end{array}$ & Experimental & $\begin{array}{l}\text { Revisa as respostas da TH17 em pacientes com SARS-CoV-2 e } \\
\text { propõe um inibidor de JAK2 aprovado pela FDA, Fedratinibe, para } \\
\text { reduzir a mortalidade de pacientes com perfis imunológicos do } \\
\text { tipo TH17. }\end{array}$ \\
\hline \multirow{3}{*}{$\begin{array}{l}\text { Anti-hipertensivos/Bloqueadores } \\
\text { de receptor da ANG II }\end{array}$} & Jurewicz M, et al., 2007 & $\begin{array}{l}\text { Human T and natural killer cells possess a } \\
\text { functional renin-angiotensin system: } \\
\text { further mechanisms of angiotensin II- } \\
\text { induced inflammation }\end{array}$ & Experimental; & $\begin{array}{l}\text { Este é o primeiro estudo a mostrar que células T e NK possuem } \\
\text { indiretamente um sistema Renina-Angiotensina e são } \\
\text { potencialmente capazes de produzir e entregar Ang II aos sítios } \\
\text { de inflamação. }\end{array}$ \\
\hline & Liu L, et al., 2009 & $\begin{array}{l}\text { Losartan, an antagonist of AT1 receptor } \\
\text { for angiotensin II, attenuates } \\
\text { lipopolysaccharide induced acute lung } \\
\text { injury in rat. Archives of biochemistry and } \\
\text { biophysics }\end{array}$ & Experimental; & $\begin{array}{l}\text { Anti-hipertensivos não são maléficos para os indivíduos que o } \\
\text { utilizam, mesmo quando infectados pelo SARS-CoV-2. }\end{array}$ \\
\hline & Bavishi C, et al., 2020 & $\begin{array}{l}\text { Coronavirus disease } 2019 \text { (COVID-19) } \\
\text { infection and renin angiotensin system } \\
\text { blockers }\end{array}$ & Revisão. & $\begin{array}{l}\text { Discute se o uso de IECA / BRA's pode reduzir a incidência ou } \\
\text { mortalidade associada a COVID-19 tanto em pacientes com } \\
\text { quanto sem indicações clínicas para esses fármacos. }\end{array}$ \\
\hline
\end{tabular}




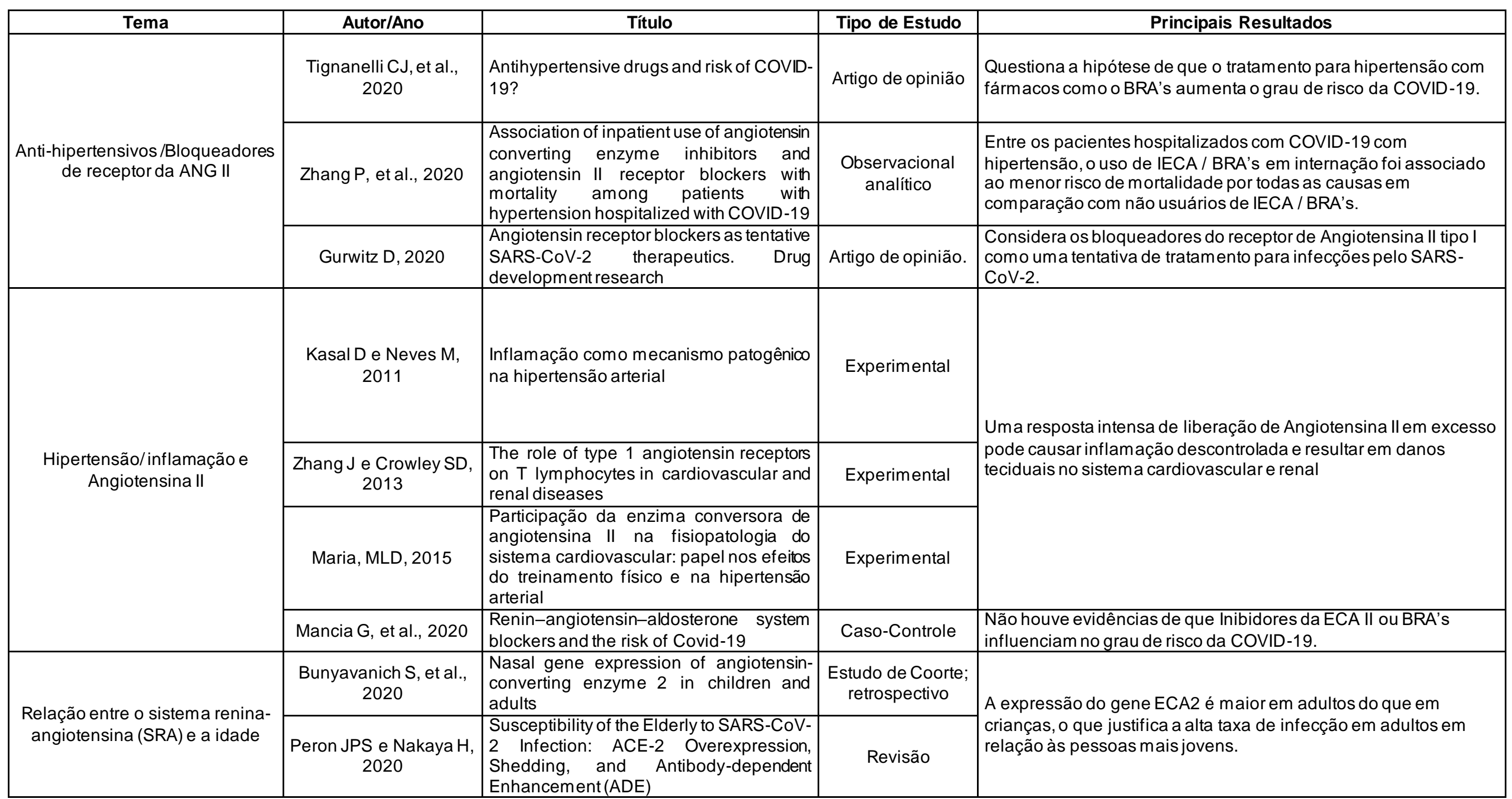

Fonte: Pampolha AFO, et al., 2021. 


\section{DISCUSSÃO}

O formato de coroa, característico dos coronavírus, é composto de proteínas denominadas "spike" cuja interação com os receptores da enzima conversora de angiotensina 2 (ECA2) promove a entrada deste vírus no sistema respiratório. Diante disso, destaca-se o fato de que a proteína "spike" do SARS-CoV-2 possui mais afinidade com os receptores ECA2 do que as proteínas do SARS-CoV 1 - vírus responsável pela primeira pandemia de SARS em 2002 e 2003 - o que justifica sua maior eficácia na disseminação entre humanos (WANG L, et al., 2019; PETROSILLO N, et al., 2020; SHANG J, et al., 2020).

Em uma visão clínica, segundo Adhikari SP, et al. (2020) as manifestações não são claras, pois os infectados variam de sintomas leves, com recuperação após uma semana e grave, expondo insuficiência respiratória progressiva devido aos danos alveolares e alguns com evolução a óbito. Em sua maioria, os sintomas relatados são: febre, mialgia, pneumonia e dispneia complicada e, de menor frequência: cefaleia, diarreia, hemoptise e coriza. No aspecto ao fator de risco para COVID-19, estudo caso-controle de $\mathrm{Yan} H$, et al. (2020) corrobora a hipertensão (55,4\%), Diabetes Mellitus (DM) (15,6\%) e doença hepática crônica (4,6\%) como comorbidades de maior prevalência em casos graves e críticos, significativamente maior no sexo masculino e faixa etária acima de 65 anos, expondo um padrão no sentido ao aumento da prevalência com a gravidade.

Em consenso, um estudo realizado por Huang C, et al. (2020) e Walls AC, et al. (2020), a maioria dos pacientes que são hospitalizados apresentam hipertensão e, como manifestações clínicas recorrentes os sintomas de febre, tosse seca, dispneia e opacidade bilateral no tórax detectado por tomografia computadorizada.

Tendo em vista as comorbidades frequentes, Yan H, et al. (2020) expuseram uma possível relação entre determinados medicamentos e a gravidade da COVID-19, cujos resultados indicam que medicamentos antidiabéticos são menos prevalentes em indivíduos com DM e sintomáticos para COVID-19 e os inibitórios da dipeptidil peptidase-4 (DDP4) e a insulina são mais prevalentes. Referente ao uso de anti-hipertensivos, os bloqueadores dos canais de cálcio (CCBs) prevaleceram e foram associados ao risco de aumento da manifestação dos sintomas da COVID-19, apesar dos mecanismos subjacentes dessa interação não serem claros e não haver evidências de que os CCBs alterem a expressão de ACE2. Ademais, não houve diferença significativa na suscetibilidade ou gravidade da COVID-19 com o uso de IECA.

Para Huang C, et al (2020), devido a elevação das citocinas induzidas pelo do SARS-CoV-2, os corticosteroides foram, frequentemente, utilizados como tratamento em uma forma de possível redução da inflamação. Contudo, evidencias atuais remetem a não influência dos corticosteroides sobre as taxas de mortalidade, e sim no retardo a eliminação viral, portanto, aumento dos riscos de gravidade da doença, logo, autoridades sanitárias não fornecem orientações e recomendações para sua utilização de forma sistêmica. $O$ mesmo perfil de gravidade foi relatado pela utilização do CCBs e betabloqueadores, onde apresentaram aumento na incidência de eventos cardiovasculares (YAN H, et al., 2020). Portanto, a essencialidade nas avaliações das ações medicamentosas ao progresso clínico dos afetados pela COVID-19, sobretudo, ao público de risco, com finalidade à diminuição das formas grave e crítica da doença e assim refletir potencialmente à saúde pública.

Segundo Casas CPR, et al. (2020), o Sars-CoV-2 expõem desafios devido à complexidade em seu sistema de replicação, o amplo espectro de inf ecção e a alta capacidade de mutação. Destarte, apesar de não haver fortes evidencias sobre eficácia no tratamento para COVID-19, atualmente, dentre as estratégias de tratamento o reposicionamento de antivirais é considerado mais eficiente e estudos alegam a potente eficácia de certo tratamento por base de antiviral de amplo espectro para infecção por MERS-CoV e SARS-CoV, como lopinavir e ritonavir, todavia, o SARS-CoV-2 é emergente, assim, estudos e ensaios clínicos randomizados em pacientes hospitalizados com infecção são necessários (CASAS CPR, et al., 2020; HUNG IF, et al., 2020; MALHANI AA, et al., 2021).

Inicialmente, os estudos traçaram esquemas de terapêutico para COVID-19 baseados na utilização de Remdesivir; Lopinavir/ Ritonavir; Lopinavir/Ritonavir com Interferon Beta-1a; e Cloroquina (CQ) ou Hidroxicloroquina $(\mathrm{HQ})$, contudo, cada esquema apresenta desafios e problemas específicos, como: 
resistência à droga, aumento na taxa de mortalidade quando realizado de forma tardia, não redução da carga viral no trato respiratório, portanto, os riscos e benefícios devem ser levados em consideração nas decisões clínicas e estudos mais aprofundados devem ser realizados (CASAS CPR, et al., 2020; CHEN J, et al., 2020; MALHANI AA, et al., 2021).

Referente ao esquema apresentando a $\mathrm{CQ}$ e $\mathrm{HQ}$, fármacos incessantemente recomendados pelo Presidente da República no Brasil, segundo Chen J, et al. (2020) e Chen Z, et al. (2020), os ensaios apresentam graus variados de viés e desenhos de estudo inadequados, distribuídos entre ensaios clínicos e estudos observacionais que, segundo definição, não são adequados para investigar a eficácia das intervenções médicas, logo, os resultados conflitantes impedem sua adoção em protocolo terapêutico.

De acordo com Rivellese F e Prediletto E (2020), o vírus atinge direta e indiretamente o Sistema ReninaAngiotensina (SRA) do sistema cardiovascular e, no processo de infecção, após a interação vírus-receptor, a enzima ECA 2 sofre downregulation e, consequentemente, observa-se um aumento significativo de angiotensina II (Ang II) - proteína de função hipertensora no SRA - no meio extracelular, uma vez que a ECA2 é a enzima responsável pela clivagem da angiotensina II em angiotensina 1-7 (Ang 1-7) e de angiotensina I (Ang I) - precursora de Ang II - em angiotensina 1-9 (Ang 1-9) (RIVELLESE F e PREDILETTO E, 2020; VADUGANATHAN M, et al., 2020).

Por outro lado, seguindo o modelo de primeiro ponto de contato para SARS-CoV-2 e o corpo humano, Bunyavanich S, et al. (2020) e Peron JPS e Nakaya H (2020), concluem que a taxa de expressão do gene da enzima ECA 2 nas vias superiores é significativamente menor em crianças quando em comparação aos adultos, expondo uma tendência linear para mudança na expressão de ACE2 com o avanço da faixa etária, o que explica a maior susceptibilidade de idosos à infecção viral em relação às pessoas mais jovens, haja vista que a maior quantidade de receptores na via nasal facilita a entrada do vírus e sua disseminação até as vias respiratórias inferiores. Dessa forma, a Figura 2 descreve de forma sucinta o processo de interferência do coronavírus no sistema renina-angiotensina e como este desencadeia em casos mais graves de SARS que podem levar o paciente a óbito e, na Figura 3, o ciclo do sistema Renina-Angiotensina.

Figura 2 - Relação entre a interação do COVID-19 com a enzima conversora de angiotensina 2 e a alteração do balanceamento no sistema renina-angiotensina (SRA).

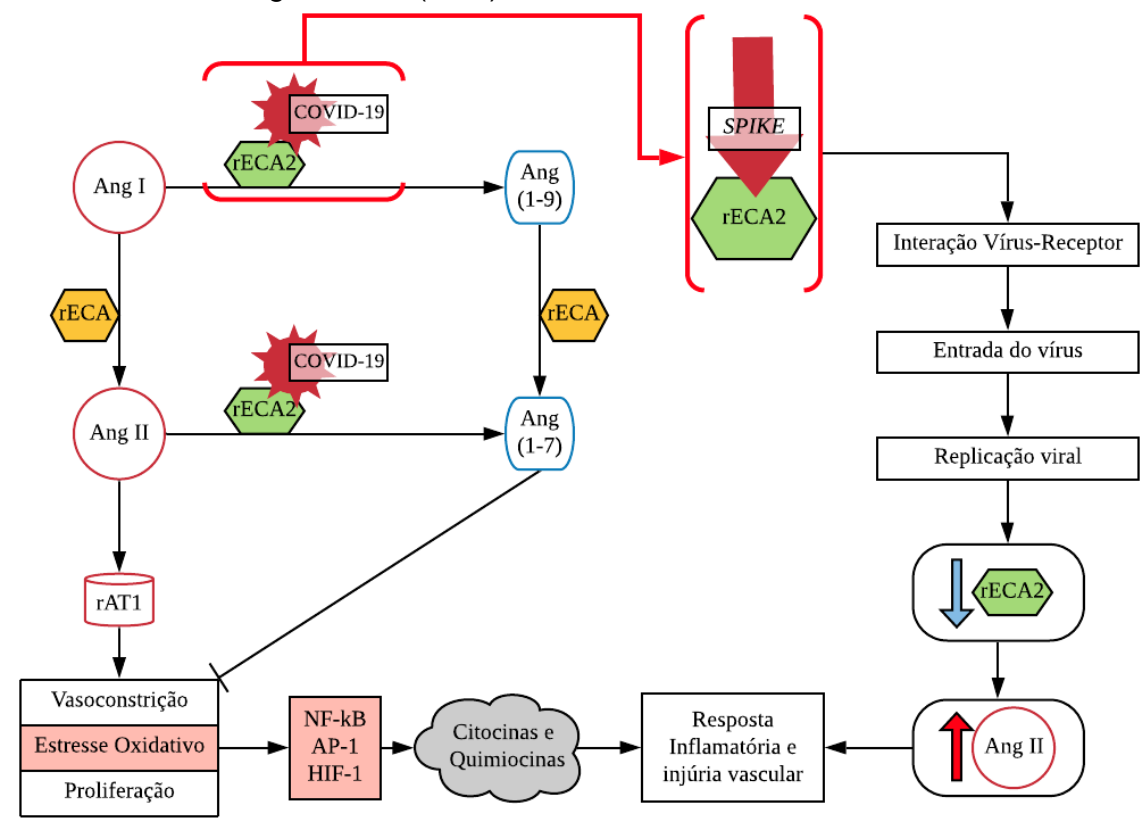

Legenda: Ang I, angiotensina I; Ang II, angiotensina II; rAT1, receptor de angiotensina do tipo 1; ECA, enzima conversora de angiotensina; ECA2, enzima conversora de angiotensina 2; Ang (1-9), angiotensina 1-7; Ang (1-9), angiotensina 1-9; NF-kB, AP-1 e HIF-1 são fatores de transcrição. Os bloqueadores de receptores de angiotensina (BRAS) atuam na proteína rECA, interrom pendo a ação da Ang II na tentativa de recuperar o balanceamento do SRA. Nota: A imagem foi construída através do @2021 Lucid Software Inc. por meio de licença livre respeitando os termos de serviços concedidos pela presente data. Fonte: Pampolha AFO, et al., 2021. Baseado em Reynolds HR, et al., 2020; Adhikari SP, et al., 2020; Fujioka S, et al., 2004; D'Ignazio L, et al., 2016. 
Figura 3 - Representação do ciclo do sistema Renina-Angiotensina.

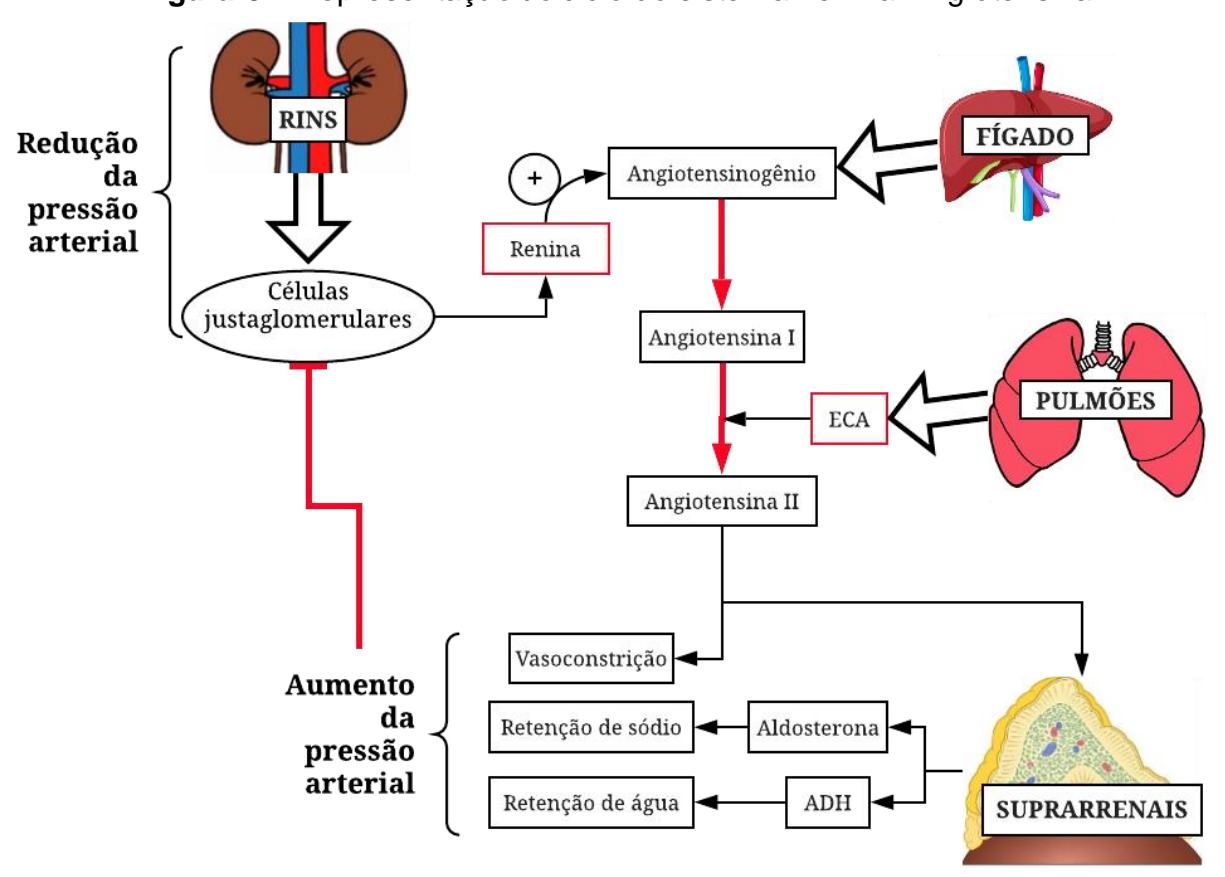

Legenda: ECA, enzima conversora de angiotensina; $A D H$, hormônio antidiurético. Nota: A imagem foi construída através do @2021 Lucid Software Inc. por meio de licença livre respeitando os termos de serviços concedidos pela presente data. Fonte: Pampolha AFO, et al., 2021. Baseado em Guyton AC e Hall JE, 2017.

Destarte, apesar dos betabloqueadores serem apontados como um potencial fármaco no tratamento da SARS é válido ressaltar que seu mecanismo de ação, diferente aos demais citados, não age diretamente no sistema renina-angiotensina, mas sim no sistema adrenérgico, logo, são formalmente contraindicados em pacientes com asma, doença pulmonar obstrutiva crônica e bloqueio atrioventricular de $2^{\circ}$ e $3^{\circ}$ graus, de acordo com o III Consenso Brasileiro de hipertensão arterial, de 1999. Dessa forma, torna-se uma alternativa não tão eficaz, uma vez que não abrange tais pacientes que são cruciais por serem considerados do grupo de risco da COVID19 (MANCIA, et al., 2020; YAN H, et al., 2020).

Como exposto, o SRA tem influência sob a pressão arterial e a função renal, logo, sendo os ECA e bloqueadores AT1R amplamente utilizados em pacientes cardíacos e hipertensos, Peron JPS e Nakaya $\mathrm{H}$ (2020) expõem os bloqueadores de receptores de angiotensina (BRA's) como uma possível alternativa farmacológica no tratamento de casos graves de SARS, pois seu uso apresenta a probabilidade de alterar a dinâmica da expressão da ACE2.

Inicialmente, era proposto que o tratamento com BRAs, em pacientes hipertensos que tivessem COVID19, fosse suspenso, uma vez que alguns estudos sugeriram que a administração desse fármaco resultava em um upregulation dos receptores de ECA2, contudo, a hipótese foi refutada (TIGNANELLI CJ, et al., 2020; MANCIA G, et al., 2020). As questões envolvendo essa relação ainda são amplamente debatidas, entretanto, sua eficácia, segurança e ausência de associação entre sua utilização e aumento na taxa de mortalidade por COVID-19 fundamenta seu ef eito protetor e sua manutenção como forma de tratamento.

Diante disso, estudos indicam que os bloqueadores de receptores de angiotensina, como a Losartana, não apresentam riscos aos pacientes e podem ser eficazes em atenuar a lesão pulmonar aguda (LPA) (LIU L, et al., 2009; GURWITZ D, 2020). Ademais, foram encontradas evidências de que os casos de pacientes hipertensos testados positivos para Sars-CoV-2 que mantiveram o tratamento com BRA's ou IECA obtiveram ef eitos benéficos contra a COVID-19 (BARAL R, et al., 2020).

Em um extenso estudo de caso-controle, foi descoberto que, dentre os indivíduos com diagnóstico de hipertensão, não houve diferença no uso de IECA's ou betabloqueadores, mas indivíduos com COVID-19 eram significativamente menos propensos a usar bloqueadores do receptor de angiotensina (BRAs) (MANCIA 
G, et al., 2020). Além disso, apesar do número de hipertensos ser mais prevalente entre os pacientes com SARS, em estado crítico em relação ao controle, o número de indivíduos com diagnóstico de COVID -19 e hipertensão que usam ARBs foi significativamente menos prevalente em comparação aos controles hipertensos (YAN H, et al., 2020).

Nesse cenário, a literatura é conflitante e, apesar de estudos relatarem interações deletérias farmacológicas dos IECA e bloqueadores AT1R à susceptibilidade por SARS-CoV-2 e/ou aumento da mortalidade, o efeito protetor quanto à injúria pulmonar causado pela COVID-19 e redução da morbimortalidade, como reflexo da diminuição da formação da angiotensina II e consequente a menor estimulação dos receptores AT1 após a entrada viral, são base para sua indicação na classe I com evidência A nas diretrizes nacionais e internacionais (PERON JPS e NAKAYA H, 2020; RIVELLESE F e PREDILETTO E, 2020).

\section{CONSIDERAÇÕES FINAIS}

Os cuidados paliativos recomendados pela Organização da Saúde (OMS) para prevenir a infecção continuam sendo as medidas mais importantes no combate à pandemia. Nesse cenário, como não há antiviral para tratar a COVID-19 e diante do surgimento das novas variantes do SARS-Cov-2 no mundo, a seguinte revisão agrega informações que indicam os benefícios do uso de BRA's no tratamento da Síndrome Respiratória Aguda Severa causada pela COVID-19. Assim, levantamos a hipótese de que por meio do bloqueio dos receptores da angiotensina, as graves lesões pulmonares que desencadeiam na SARS seriam minimizadas consideravelmente, haja vista que o sistema renina-angiotensina estimula o sistema imune que desencadia uma cascata inf lamatória que resulta na cytokine storm e posteriormente nos danos teciduais que, em muitos casos, são irreversíveis ou deixam sequelas. Contudo, a realização de estudos experimentais com o objetivo principal de avaliar o fármaco é necessária para comprovar a eficácia do fármaco.

\section{REFERÊNCIAS}

1. ADHIKARI SP, et al. Epidemiology, causes, clinical manifestation and diagnosis, prevention and control of coronavinus disease (COVID-19) during the early outbreak period: a scoping review. Infectious diseases of poverty, 2020;9(1): 112.

2. BARAL R, et al. Effect of renin-angiotensin-aldosterone system inhibitors in patients with COVID-19: a systematic review and meta-analysis of 28,872 patients. Current atherosclerosis reports, 2020; 22(10):1-9.

3. BAVISHI C, et al. Coronavirus disease 2019 (COVID-19) infection and renin angiotensin system blockers. JAMA cardiology, 2020;5(7): 745-747.

4. BRANDÃO SCS, et al. COVID-19 grave: entenda o papel da imunidade, do endotélio e da coagulação na prática clínica. J Vasc Bras, 2020; 19.

5. BUNYAVANICH S, et al. Nasal gene expression of angiotensin-converting enzyme 2 in children and adults. Jama, 2020;323(23):2427-2429.

6. CASAS CPR, et al. Avaliação de tecnologias em saúde: tensões metodológicas durante a pandemia de Covid-19. Estudos Avançados, 2020;34(99).

7. CARVALHO CA, et al. In Vitro Inhibition of SARS-CoV-2 Infection by Bovine Lactoferrin. bioRxiv, $2020 ; 13$.

8. CHEN J, et al. A pilot study of hydroxychloroquine in treatment of patients with moderate Covid-19. Zhejiang Da Xue Xue Bao Yi Xue Ban, 2020; 49(2):215-219.

9. CHEN Z, et al. Efficacy of hydroxychloroquine in patients with COVID-19: results of a randomized clinical trial. medRxiv, $2020 ; 17$.

10. D'IGNAZIO L, et al. NF-KB and HIF crosstalk in immune responses. The FEBS journal, 2016;283(3):413-424.

11. FUJIOKA S, et al. NF-KB and AP-1 connection:mechanism of NF-kB-dependent regulation of AP-1 activity. Molecular and cellular biology, 2004;24(17): 7806-7819.

12. GURWITZ D. Angiotensin receptor blockers as tentative SARS-CoV-2 therapeutics. Drug development research, 2020; 81(5): 537-540.

13. GUYTON AC, HALL JE. Tratado de Fisiologia Médica. Editora Elsevier. 13ae ed., 2017. 232p.

14. HUNG IF, et al. Triple combination of interferon beta-1b, lopinavir-ritonavir, and ribavirin in the treatment of patients admitted to hospital with Covid-19: an open-label, randomised, phase 2 trial. Lancet, 2020; 395(10238):1695-704.

15. HUANG C, et al. Clinical features of patients infected with 2019 novel coronavirus in Wuhan, China. The lancet, 2020; 395(10223): 497-506.

16. JUREWICZ $M$, et al. Human $T$ and natural killer cells possess a functional renin-angiotensin system: further mechanisms of angiotensin ii-induced inflammation. Journal of the American Society of Nephrology, 2007; 18(4): 1093-1102. 
17. KASAL D, NEVES M. Inflamação como mecanismo patogênico na hipertensão arterial. Revista Hospital Universitário Pedro Ernesto, 2011;10(3).

18. LANA RM, et al. Emergência do novo coronavírus (SARS-CoV-2) e o papel de uma vigilância nacional em saúde oportuna e efetiva. Cadernos de Saúde Pública, 2020;36.

19. LIU L, et al. Losartan, an antagonist of AT1 receptor for angiotensin II, attenuates lipopolysaccharide-induced acute lung injury in rat. Archives of biochemistry and biophysics, 2009;481(1):131-136.

20. MALHANI AA, et al. Combination of (interferon beta-1b, lopinavir/ritonavir and ribavirin) versus favipiravir in hospitalized patients with non-critical COVID-19: A cohort study. PLoS One, 2021;10: 16(6).

21. MANCIA G, et al. Renin-angiotensin-aldosterone system blockers and the risk of Covid-19. New England Journal of Medicine, 2020;382: 2431-2440.

22. MARIA MLD. Participação da enzima conversora de angiotensina 2 na fisiopatologia do sistema cardiovascular:papel nos efeitos do treinamento físico e na hipertensão arterial. Dissertação (Doutorado em Biologia Celular) - Instituto de Ciências Biológicas. Universidade do Estado de Minas Gerais, Belo Horizonte, 2015.

23. MEO SA, et al. Novel coronavirus 2019-nCoV: prevalence, biological and clinical characteristics comparison with SARS-CoV and MERS-CoV. Eur Rev Med Pharmacol Sci, 2020;24(4): 2012-2019.

24. MINISTÉRIO DA SAÚDE. Painel de casos de doença pelo coronavírus 2019 (COVID-19) no Brasil pelo Ministério da Saúde, Brasil, 2021. Disponível em: https://covid.saude.gov.br/. Acesso em:02 jul. 2021.

25. PERON JPS, NAKAYA H. Susceptibility of the Elderly to SARS-CoV-2 Infection: ACE-2 Overexpression, Shedding, and Antibody-dependent Enhancement (ADE). Clinics, 2020;75.

26. PETROSILLO N, et al. COVID-19, SARS and MERS: are they closely related?. Clinical Microbiology and Infection, 2020;26(6): 729-734.

27. REYNOLDS HR, et al. Renin-angiotensin-aldosterone system inhibitors and risk of Covid-19. New England Joumal of Medicine, 2020;382: 2441-2448.

28. RIVELLESE F, PREDILETTO E. ACE2 at the centre of COVID-19 from paucisymptomatic infections to severe pneumonia. Autoimmunity Reviews, 2020; 19(6): 102536.

29. SHANG J, et al. Structure of mouse coronavirus spike protein complexed with receptor reveals mechanism for viral entry. PLoS pathogens, 2020;16(3).

30. TIGNANELLI CJ, et al. Antihypertensive drugs and risk of COVID-19?. The Lancet Respiratory Medicine, 2020;8(5): 30-31.

31. TISONCIK JR, et al. Into the eye of the cytokine storm. Microbiology and Molecular Biology Reviews, 2012; 76(1): 1632.

32. UZUNIAN A. Coronavírus SARS-CoV-2 e Covid-19. J Bras Patol Med Lab, 2020; 56: 1-4.

33. VADUGANATHAN M, et al. Renin-angiotensin-aldosterone system inhibitors in patients with Covid-19. New England Journal of Medicine, 2020;382(17): 1653-1659.

34. WALLS AC, et al. Structure, function, and antigenicity of the SARS-CoV-2 spike glycoprotein. Cell. 2020; 181(2): 281 292.

35. WANG L, et al. Review of the 2019 novel coronavirus (SARS-CoV-2) based on current. International Journal of Antimicrobial Agents, 2019;55(6).

36.WU D, YANG XO. TH17 responses in cytokine storm of COVID-19: An emerging target of JAK2 inhibitor Fedratinib. Journal of Microbiology, Im munology and Infection, 2020;53(3):368-370.

37. YAN H, et al. Role of Drugs used for chronic disease management on Susceptibility and Severity of COVID -19: A Large Case-Control Study. Clinical Pharmacology \& Therapeutics, 2020;108(6): 1185-1194.

38. YE Q, et al. The pathogenesis and treatment of the "Cytokine Storm" in COVID-19. Journal of infection, 2020; 80(6): 607-613.

39. ZHANG J, CROWLEY SD. The role of type 1 angiotensin receptors on $T$ lymphocytes in cardiovascular and renal diseases. Current hypertension reports. 2013;15(1): 39-46.

40. ZHANG P, et al. Association of inpatient use of angiotensin converting enzyme inhibitors and angiotensin II receptor blockers with mortality among patients with hypertension hospitalized with COVID-19. Circulation research, 2020; 126(12):1671-1681. 